\title{
Topology optimization and experimental verification of compact E-plane waveguide filters
}

Nielsen, Daniel Gert; Pedersen, Søren Damgaard; Zhurbenko, Vitaliy; Johansen, Villads Egede; Sigmund, Ole; Aage, Niels

\section{Published in:}

Microwave and Optical Technology Letters

Link to article, DOI:

10.1002/mop.31741

Publication date:

2019

Document Version

Peer reviewed version

Link back to DTU Orbit

Citation (APA):

Nielsen, D. G., Pedersen, S. D., Zhurbenko, V., Johansen, V. E., Sigmund, O., \& Aage, N. (2019). Topology optimization and experimental verification of compact E-plane waveguide filters. Microwave and Optical Technology Letters, 61(5), 1208-1215. https://doi.org/10.1002/mop.31741

\section{General rights}

Copyright and moral rights for the publications made accessible in the public portal are retained by the authors and/or other copyright owners and it is a condition of accessing publications that users recognise and abide by the legal requirements associated with these rights.

- Users may download and print one copy of any publication from the public portal for the purpose of private study or research.

- You may not further distribute the material or use it for any profit-making activity or commercial gain

- You may freely distribute the URL identifying the publication in the public portal 


\title{
Topology Optimization of Compact E-Plane Waveguide Filters
}

\author{
Daniel Gert Nielsen ${ }^{1},{ }^{4}$, Søren Damgaard Pedersen ${ }^{2}$, Vitaliy Zhurbenko ${ }^{1}$, Member, IEEE, Villads Egede Johansen ${ }^{3}$, \\ Ole Sigmund ${ }^{2}$, and Niels Aage $^{2}, 4$ \\ ${ }^{1}$ Department of Electrical Engineering, Technical University of Denmark, Kgs. Lyngby DK-2800 \\ ${ }^{2}$ Department of Mechanical Engineering, Technical University of Denmark, Kgs. Lyngby DK-2800 \\ ${ }^{3}$ Department of Chemistry, University of Cambridge, Lensfield Rd., Cambridge, CB2 IEW, UK \\ ${ }^{4}$ Centre for Acoustic-Mechanical Micro Systems, Technical University of Denmark, Kgs. Lyngby DK-2800
}

\begin{abstract}
This paper presents a numerical method to design compact E-plane waveguide filters. The key feature of the proposed method is that the conducting layout of the filters is automatically determined based on a desired filter characteristic. This extreme level of design freedom is obtained using the numerical optimization technique called topology optimization. The filters are complex in shape but easy to mass-produce with low-cost conventional methods. The method is verified experimentally by measurement of responses similar to the numerical predictions.
\end{abstract}

Index Terms-Compact Filters, E-Plane Filters, Rectangular Waveguide, Topology Optimization

\section{INTRODUCTION}

W AVEGUIDE filters are indispensable assets in high frequency systems such as radars and satellites [1]. Several types of waveguide filters exist where E-plane filters have been widely used in millimetre wave applications since their introduction by Konishi and Uenakada [2], due to their compact size, low-cost, mass produceability and low loss.

Rising demands to performance, frequency selectivity, size and weight of waveguide filters mean that they are constantly being improved. Metallic insert filters are subject to such improvements. One method is to place transmission zeroes at discrete frequencies [3], [4]. Goussetis et al. [5] also utilize this method and a compact filter is obtained by cross coupling of resonators. This filter is $35 \mathrm{~mm}$ long, corresponding to $1.05 \lambda_{g}$, where $\lambda_{g}$ is the wavelength of the center frequency in the passband in a rectangular waveguide. All of the above mentioned filters are based on short-circuiting the waveguide walls. Lopez-Villarroya et al. [6] presents a waveguide filter with cshaped resonators which are decoupled from the waveguide walls.

Though E-plane filters have several advantages in terms of easy manufacturing, The European Space Agency (ESA) has recently produced cavity filters with extremely high design freedom enabled by metallic 3D printing of the entire waveguide including the waveguide filter [7]. It would therefore be desirable to have a design method with geomtric freedom similar to that of ESA's cavity filters, but maintaining the low costs of conventional E-plane filters.

Computer-based optimization of waveguide bandpass filters is an established research topic. Bornemann et al. [8] present an evolution based optimization strategy for ladder-like filter structures. Aydogan et al. [9] optimize the placement and thickness of dielectric slabs placed in a waveguide with a Levenberg-Marquadt optimization scheme. Budimir and Goussetis [10] optimize asymmetrical ridged-waveguide bandpass filters with respect to the Chebyvev criterion, where the height of the ridges are the variables. Ouedraogo et al. [11] present pixelated filter inserts for bandstop waveguide filters, optimized with a genetic algorithm.

Gradient-based topology optimization has proven to be usefull for optimizing aerospace applications such as wings [12], structural, dynamic and fluid mechanics [13] and for various types of electromagnetic problems. For conductor based material distribution problems in electromagnetics, most effort has been put in the design of different types of antennas, see e.g. [14]-[16]. Diaz and Sigmund [17] propose a method for designing metamaterials with negative permeability. Hassan et al. [18] maximize the matching between a coaxial cable and rectangular waveguide. Khalil et al. [19] optimize the shape of dielectric microwave components for dual-mode waveguide filters. Recently, gradient based topology optimization has been demonstrated for the optimization of bandpass filters [20].

The procedure proposed in [20] is extended here, applied to practical problems and verified experimentally. The designs are etched on Printed Circuit Boards (PCB)s. The design method empowers complete design freedom and thereby the ability to tailor the filters to distinct frequencies ranges, thus the proposed method can be utilized to create various filter types, such as bandpass, bandstop, highpass filters or filters with several operating bands.

The remainder of the paper is organized as follows; first, we present the filter design methodology along with the governing physical model. Then a description of the fabrication process and the experimental setup is given. Finally, we present a number of optimized filters and discuss the achieved characteristics.

\section{DESIGN THEORY}

This section serves as a brief overview of the overall design method. The main goal in [20] was to devise a design 


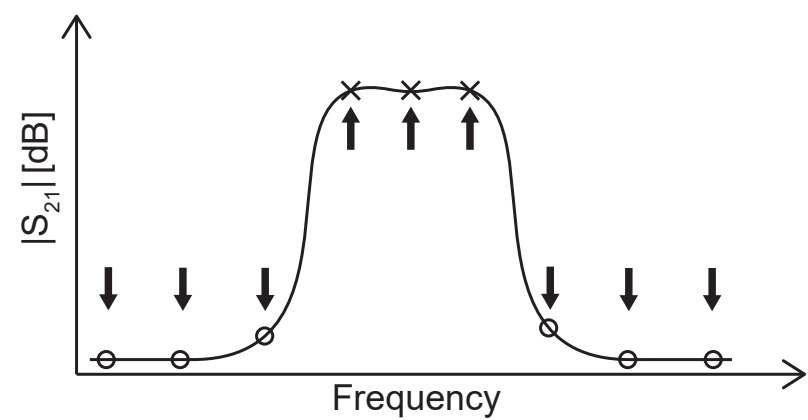

Fig. 1. Schematic illustration of the optimization of a bandpass filter at discrete frequencies (Phase 2). The upward arrows on the targeted frequencies indicate that these are to be maximized, and the downward arrows indicate the targeted frequencies to be minimized.

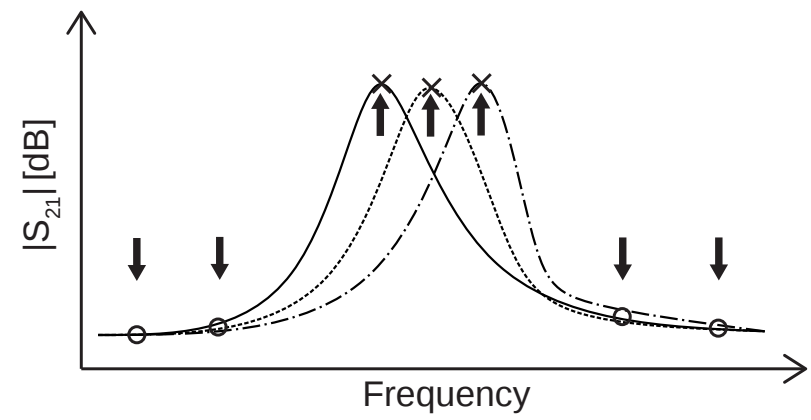

Fig. 2. Schematic illustration of the optimization of three structures that have full transmission in a finite frequency range (Phase 1).

methodology capable of realizing optimized filter inserts with specified filter characteristics. A key point was that the design should be obtained with no a priori knowledge of the layout of the filter insert itself. That is, the optimization starts with a clean PCB slab in a waveguide, and then automatically determines the spatial layout of the conducting material which results in the desired filter characteristics. This unusual degree of design freedom is achieved using the numerical optimization technique called topology optimization (TO). TO is an iterative numerical method used to determine optimized material distributions in a multitude of different physical disciplines such as structural engineering, optics, acoustics and fluid dynamics to name but a few [13].

The TO method in [20] is most easily described using an example of a bandpass filter characteristics shown in Figure 1. Using a variable number of discrete target frequencies; here a total of nine, marked with crosses and circles; the aim of the optimization is to maximize the transmission in the passband (upward arrows) while minimizing the out-of-band transmission (downward arrows).

This characteristic can be obtained by the formulation of a general max-min optimization problem, which also serves as an objective function used to evaluate the performance of the filter

$\Phi=\max \left[\min \left\{\min _{j \in \mathbb{I}_{f}}\left\{\left|S_{21}\left(\omega_{j}\right)\right|\right\}, \min _{j \in \mathbb{I}_{z}}\left\{1-\left|S_{21}\left(\omega_{j}\right)\right|\right\}\right\}\right]$,

where $\mathbb{I}_{f}$ and $\mathbb{I}_{z}$ correspond to full and zero transmission (crosses and circles in Figure 1), respectively, and $\Phi=1$ corresponds to the situation where all the scattering parameters of all the discrete frequencies have reached the optimum. The situation $\Phi=0$ is present when one or more of the scattering parameters act entirely opposite of the intended.

However, the findings in [20] show that obtaining functional filter designs with sharp rejection skirts is a challenging optimization problem. Therefore the authors propose a two phase optimization scheme which results in a more robust design methodology. The idea is to first design a series of structures with full transmittance within the operating band of the final filter. The phase 1 design problem is illustrated in Figure 4(top), which shows that three separate structures with maximum transmittance are to be determined, that is, each structure corresponds to one target frequency to be maximized as shown in Figure 1. The series of structures can be chosen arbitrarily, but one should keep in mind that the PCB slab must have enough space to contain a number of non-overlapping design domains. A rule of thumb is to include at least two frequencies (and domains) such that the upper and lower limit of the bandpass region are included.

Having completed the design of three structures with full transmittance in a finite frequency range the next phase is to design the actual filter characteristics. That is, combining these structures into a single PCB slab, see Figure 3. We allow the optimizer to simultaneous maximize and minimize the relevant discrete frequencies as seen on Figure 1, note that the target frequencies to be maximized in phase 2 are the same as phase 1 .

The complete design approach can be summarized as follows:

P1 Optimize a number of structures with full transmittance separated in both space and frequency. No initial design is needed c.f Figure 3(top).

P2 Combine these structures into a single PCB slab and use this as an initial guess for the actual filter characteristic optimization, c.f. Figure 3 (bottom).

The solution to the optimization problem is obtained by the design problem cast as two smooth mathematical programmes, which can be solved efficiently with gradient based methods. In all of the presented results we use the Method of Moving Asymptotes [21], [22].

\section{A. The waveguide model}

Before presenting the model waveguide problem and the Maxwell solver, we note that the design problem solved in this work has one major difference to [20]. In this work the filter insert is prohibited from touching the waveguide walls. This is done to make the fabrication process more robust, since it is often not easy to provide a good contact between the waveguide and the metal of the insert. The quality of this contact has a big influence on the performance of the filter. Thus, to enhance the practical applicability of the proposed design methodology, we add a small clearance $\left(h_{f}\right.$ in Figure 4) between conductor and waveguide walls.

Throughout this work we use a waveguide model as shown in Figure 4. For the phase 1 optimization the design domain is split into three subdomains $\left(\Omega_{1}, \Omega_{2}\right.$ and $\left.\Omega_{3}\right)$ which are 


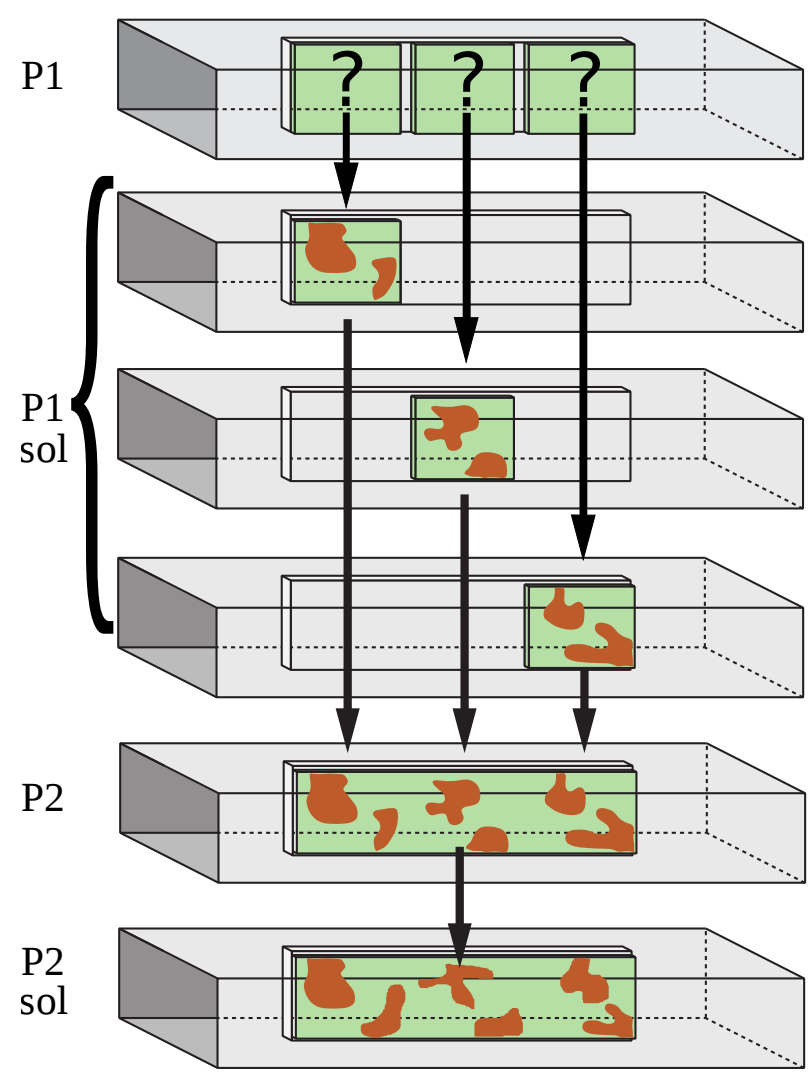

Fig. 3. Schematic illustration of the transition from a PCB slab subdivided into a number of independent optimization problems to obtain phase 1 results. These results are collected into a single domain as the initial guess for the phase 2 optimization problem. The bottom illustration is the results of the phase 2 optimization, which is the final filter design.

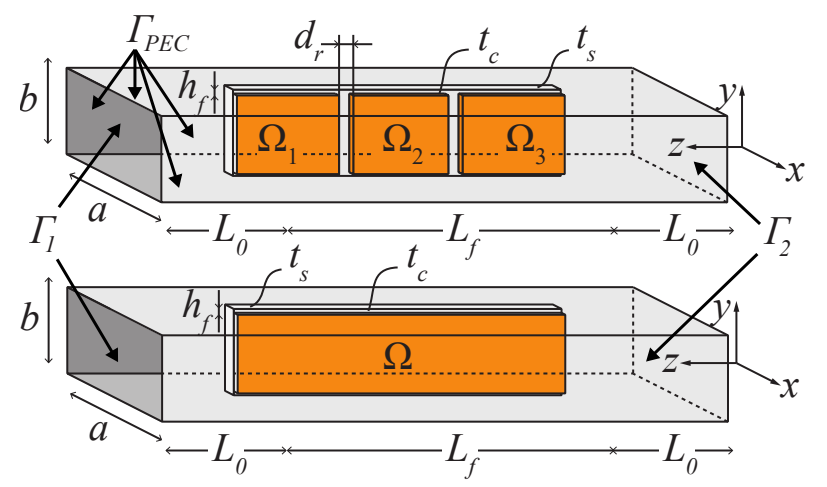

Fig. 4. Design domains of phase 1 (top) and phase 2 (bottom). Dimensions are $a=22.86 \mathrm{~mm}, b=10.16 \mathrm{~mm}, L_{0}=5.00 \mathrm{~mm}, L_{f}=32.59 \mathrm{~mm}$, $h_{f}=0.50 \mathrm{~mm}, d_{r}=1 \mathrm{~mm}, t_{c}=0.035 \mathrm{~mm}$ and $t_{s}=0.79 \mathrm{~mm}$. The filter insert is a Rogers duroid 6002 and the operating frequency range is between 8.0 and $12.0 \mathrm{GHz}$.

collected into a single domain $(\Omega)$ for phase 2, c.f. Figure 3 and 4. The operating range for the filters in this work is 8.0 to $12.0 \mathrm{GHz}$, corresponding to the rectangular waveguide standard WR-90. The filters are compact with a length of 32.6 mm corresponding to $1.1 \lambda_{g}$ when the center frequency is 10.3 $\mathrm{GHz}$.

\section{B. Numerical setup}

The waveguide model consist of a two-port system, as shown in Figure 4 with a $\mathrm{TE}_{10}$ wave incident from the left. To obtain the numerical prediction of the waveguide with the filter inserted we solve Maxwell's equation cast in the frequency domain, i.e.

$$
\nabla \times\left(\mu_{r}^{-1} \nabla \times \mathbf{E}\right)-k_{0}^{2}\left(\epsilon_{r}-j \frac{\sigma(\mathbf{x})}{\omega \epsilon_{0}}\right) \mathbf{E}=0 \text { in } \Omega,
$$

where $\mu_{r}$ is the relative permeability, $\epsilon_{r}$ is the relative permittivity, $\sigma(\mathbf{x})$ is the spatially varying conductivity, $\omega$ is the frequency of the microwave signals, $\mathbf{E}$ is the electric field, $k_{0}=\omega \sqrt{\epsilon_{0} \mu_{0}}$ is the free space wavenumber, $\epsilon_{0}$ and $\mu_{0}$ are the permittivity and permeability of vacuum, respectively. We note that the spatial variation in conductivity is introduced as a design variable for the optimization. That is, the task of the optimization process is to determine where to place conductive material and where not to. For details on the optimization method the reader is referred to [20] and [23].

The waveguide walls are modelled as Perfect Electric Conductors (PEC)s

$$
\mathbf{n} \times \mathbf{E}=\mathbf{0} \text { on } \Gamma_{P E C},
$$

where $\mathbf{n}$ is an outward normal vector to the respective surfaces. The port conditions are introduced using the waveguide port boundary conditions [24]. In the operating range of the filter, only the $\mathrm{TE}_{10}$ mode exists and therefore the boundary condition is as follows

$$
\mathbf{e}_{10}^{\mathrm{TE}}(\mathbf{x})=-\sqrt{\frac{2}{a b}}\left\{\begin{array}{c}
0 \\
\sin (\pi x / a) \\
0
\end{array}\right\} .
$$

The incident field $\mathbf{E}_{\text {inc }}$ is applied at port $\Gamma_{1}$, which gives rise to the following boundary conditions

$$
\begin{aligned}
& \mathbf{n} \times \nabla \times \mathbf{E}=j \gamma \mathbf{e}_{10}^{T E} \cdot \int_{\Gamma_{1}} \mathbf{e}_{10}^{T E} \cdot \mathbf{E} \mathrm{d} \Gamma-2 j \gamma \mathbf{e}_{10}^{T E} \text { on } \Gamma_{1}, \\
& \mathbf{n} \times \nabla \times \mathbf{E}=j \gamma \mathbf{e}_{10}^{T E} \cdot \int_{\Gamma_{2}} \mathbf{e}_{10}^{T E} \cdot \mathbf{E} \mathrm{d} \Gamma \quad \text { on } \Gamma_{2},
\end{aligned}
$$

where $\gamma=\sqrt{k_{z}^{2}-k_{0}^{2}}$ defines the propagation constant, $k_{z}=$ $\pi / a$ is the wave cutoff number, and we note that only port 1 is subject to the incident field.

The boundary-value problem is solved by the finite element method using curl-conforming elements [24]. The geometries are generated and discretized using CUBIT [25] and subsequently partitioned by METIS [26]. The indefinite system of equations is then solved using MUMPS [27], [28] using an inhouse parallel code framework written in C++ using MPI [22]. The in-house code is validated using the commercial software Comsol Multiphysics 5.3. The two models are coherent when modelling a waveguide with an inserted filter.

\section{NUMERICAL AND EXPERIMENTAL RESULTS}

The filters are manufactured by photolithography on Rogers Duroid 6002 PCB with $\epsilon_{r}=2.94$ and a thickness of $0,76 \mathrm{~mm}$, which ensures that the filter is mechanically stable. Figure 5 shows a filter design with a 5 euro cent for scale. The manufacturing tolerances are estimated to be around $0.2 \mathrm{~mm}$. 


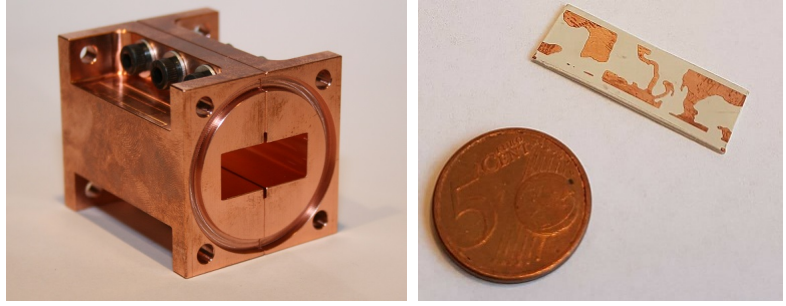

Fig. 5. Two-piece copper waveguide used in the experimental work and a filter design with a 5 euro cent for scale.

The performance of the optimized filters were tested experimentally on a Vector Network Analyzer (VNA). We fabricated a $50 \mathrm{~mm}$ long WR-90 rectangular waveguide in copper with an insertion slot to slide in the PCBs without disassembling the waveguide (Figure 5).

\section{A. Physical verification of numerical model}

Several experiments showed a discrepancy from the numerical results, where the experimental results were frequency up-shifted $0.3-0.5 \mathrm{GHz}$. To account for this, the permittivity of the PCB substrate from the data sheet $\left(\epsilon_{r}=2.94\right)$ was corrected, such that the numerical and experimental results better correlate. It was found that changing $\epsilon_{r}=2.45$ in the numerical model provides a good fit in all cases. We have investigated this issue further by manufacturing over and under etched designs, numerically we investigated mesh dependency, thickness of the conducting layout and over/under etching. We found that these parameters influence the scattering parameters, however, not as significantly as the observed up-shift of $0.3-0.5 \mathrm{GHz}$.

\section{B. Bandpass filter}

Here we will elaborate on how a bandpass filter is designed with the proposed method and the performance of this will be evaluated. The three structures with full transmittance in a finite frequency range from the phase 1 optimization were tailored to serve as starting guess for the phase 2 optimization of a bandpass filter with center frequency of $10.3 \mathrm{GHz}$. The designs and performances of the three structures are shown in Figure 6. At the start of phase 2 the initial guess from phase 1 is "smeared out", in-order to increase the design freedom and to avoid being stuck in a local minimum. The phase 2 optimization is implemented such that it maximizes transmission for the three distinct frequencies that were also maximized in phase 1 . The 6 frequencies to be minimized are evenly distributed in the stopband. The manufactured final PCB design is shown in Figure 7.

Numerically obtained S-parameters for the bandpass filter are compared to measured S-parameters in Figure 8. The green circles indicate the frequencies that were maximized during optimization, and the red circles indicate the ones that were minimized. Apart from a minor frequency shift, good agreement between the numerical and experimental results have been obtained with a measured insertionloss (IL) of 0.6 $\mathrm{dB}$ and a steep cut-of between the passband and the stopband.

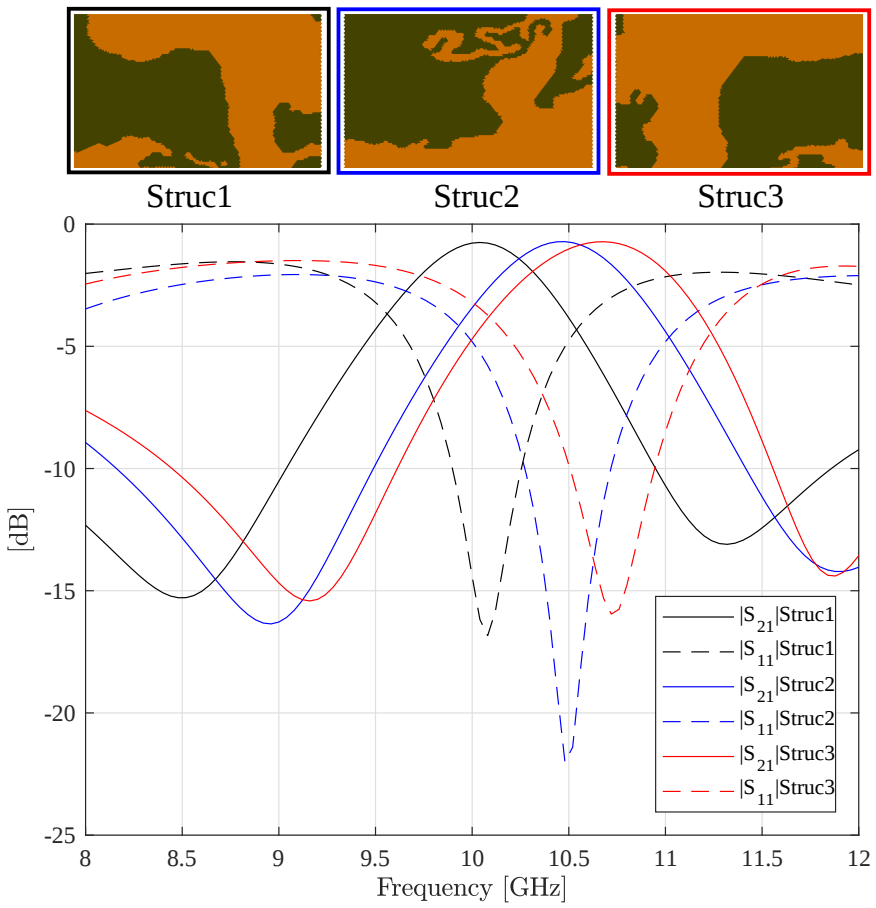

Fig. 6. S-parameters and shape of the structures originating from a phase 1 optimization. Orange areas are the copper features, in the dark green areas no conducting material is present.

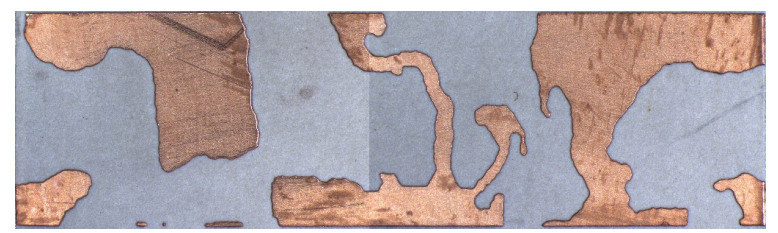

Fig. 7. Micrograph of the bandpass filter insert. The difference in lighting is due to the merging of two images. Note that the $0.5 \mathrm{~mm}$ blank substrate between the copper features and the waveguide walls is not included here.

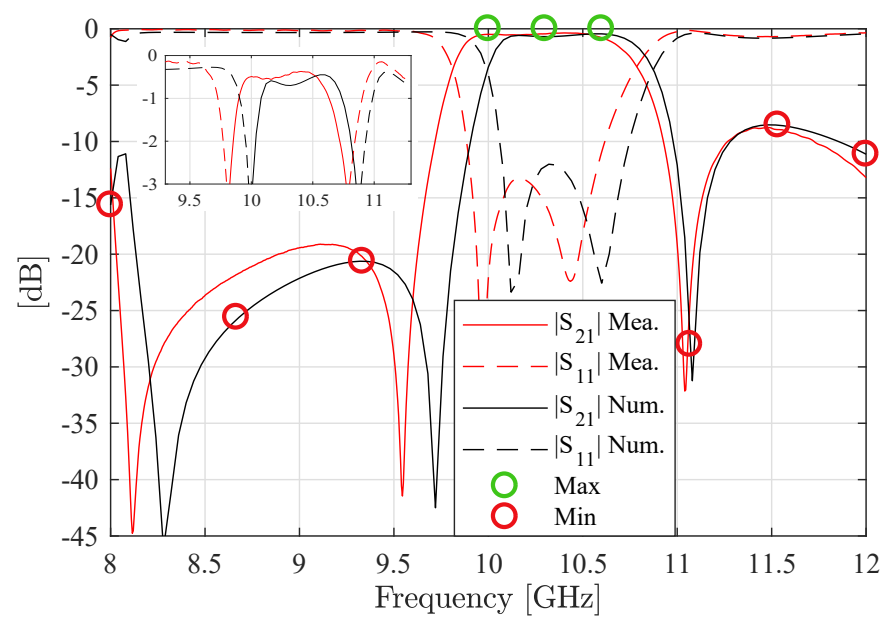

Fig. 8. Comparison between numerical and experimental results for the bandpass filter in Figure 7. Red curves are the measured results, and the black curves are the frequency response from the numerical model. The circles indicate the discrete frequencies used in the optimization. Maximum measured $\mathrm{IL}$ in the bandpass region is $0.6 \mathrm{~dB}$ and minimum rejection outside of the passband is $8.8 \mathrm{~dB}$. 


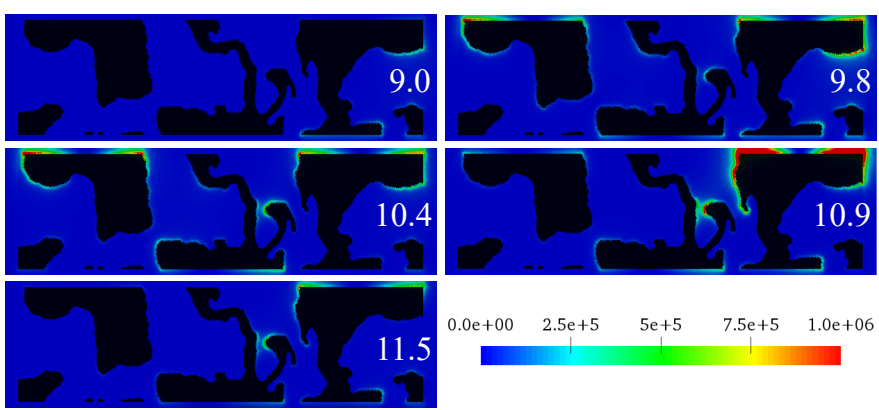

Fig. 9. Visualization of the time-average electric energy [J] at different frequencies, with an overlay of the design from Figure 7 (black domains). The wave propagates from left to right. The frequencies in each figure are in GHz. The time-average electric energy is captured in the $y z$-plane at $x=$ $-0.4 \mathrm{~mm}$. Notice that some of the field intensities exceed the bounds of $10^{6}$ $\mathrm{J}$ of the colorbar.

The conducting layout seen in Figure 7 takes organic shapes, which is different from conventional E-plane filters, and it is as such difficult to comprehend how the filter works. The design is therefore not easily obtainable through traditional methods such as parameter optimization based on fixed geometries. The time-average electric energy [29] for five frequencies located within and around the bandpass region are shown in Figure 9. This serves to show that different parts of the filter are active depending on the frequency. For example, at $9.0 \mathrm{GHz}$, only the rightmost part of the filter is active. In the bandpass region $(10.4 \mathrm{GHz})$ most of the filter is active. For the roll-offs (9.8 $\mathrm{GHz}, 10.9 \mathrm{GHz}$ ) the field intensities have some similarities we note that generally the rightmost part of the filter is more active at $10.9 \mathrm{GHz}$ and the leftmost part at $9.8 \mathrm{GHz}$. At 11.5 $\mathrm{GHz}$, the field intensities take lower values, which is also the case at $9.00 \mathrm{GHz}$, where we observe that the leftmost part of the filter is not active. A general observation is that the electric field concentrates in the gap between the copper features and the waveguide walls. This is especially pronounced for the roll-offs and the bandpass region.

\section{Filter obtained with projection methods}

At the cost of additional computational power, the filter presented in Figure 8 can be improved with a numerical projection method based on the Heaviside function. The method is thoroughly described in [30]. The phase 2 optimization is extended with the projection method applied. Figure 10 shows minor, but distinct, changes in shape and topology compared to Figure 7. Figure 11 shows that the out-of-band performance is significantly enhanced with a minimum measured rejection of $16.0 \mathrm{~dB}$, while the steep cut-off between the passband and the stopband is maintained. The cost of this is a larger IL of $1.5 \mathrm{~dB}$ for the manufactured design, wheras the numerical model predicts an IL of $1.0 \mathrm{~dB}$. Good correlation is obtained between the numerical model and the manufactured design, especially in the bandpass region and the roll-offs. This extra step demonstrates that the design approach can be tweaked in different directions depending on criteria and purpose.

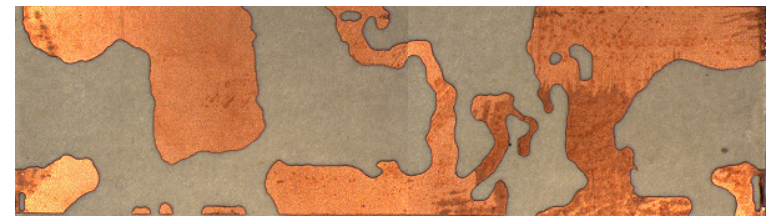

Fig. 10. Micrograph of the bandpass filter insert obtained with projection methods.

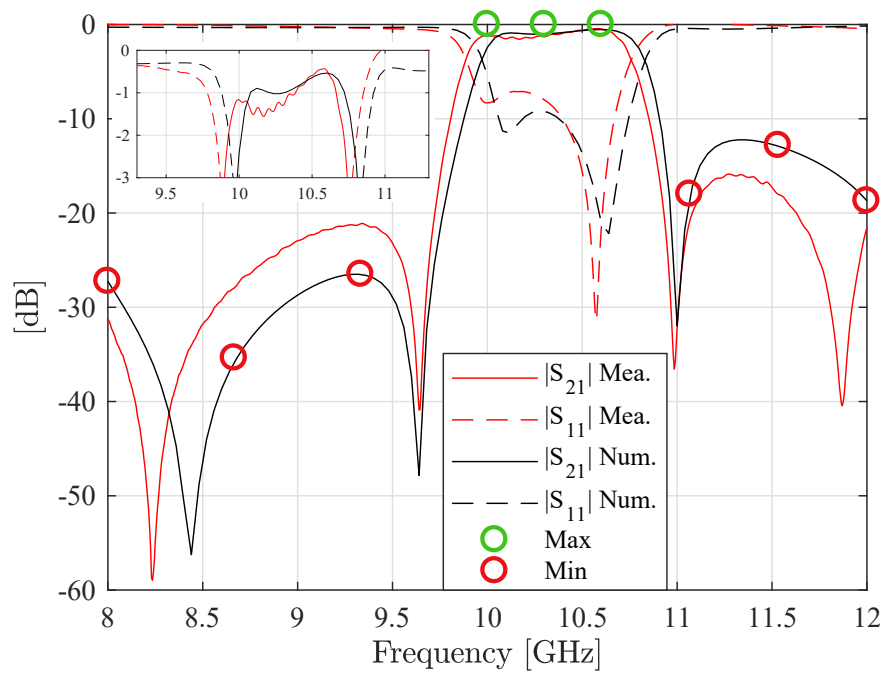

Fig. 11. Comparison between numerical and experimental results for the bandpass filter obtained with projection methods (Figure 10). Maximum measured IL in the bandpass region is $1.5 \mathrm{~dB}$ and minimum rejection outside of the passband is $16.0 \mathrm{~dB}$.

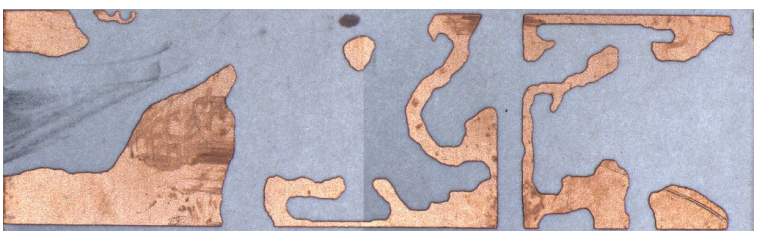

Fig. 12. Micrograph of the highpass filter insert.

\section{Highpass filter}

To demonstrate the versatility of the approach, a highpass filter with a maximum insertion loss of $0.8 \mathrm{~dB}$ was also designed (Figure 12).

The passband ranges from $10.4 \mathrm{GHz}$ to $12.0 \mathrm{GHz}$ and a steep cut-off between the passband and stopband is obtained, as shown in Figure 13. The numerical model predicts a usable highpass filter, however, this is corrupted by a reflection situated just below $12 \mathrm{GHz}$ in the measured results. This is due to the tolerances on the manufactured design, a small resonance is situated just above $12 \mathrm{GHz}$ which is slightly shifted down for the manufactured design.

\section{CONCLUSION}

E-plane filters are successfully designed with gradient based topology optimization. The resulting designs are manufactured by photolithography on Rogers Duroid 6002 and the experimental results validate the numerical model. We see that the out-of-band performance can be increased using 


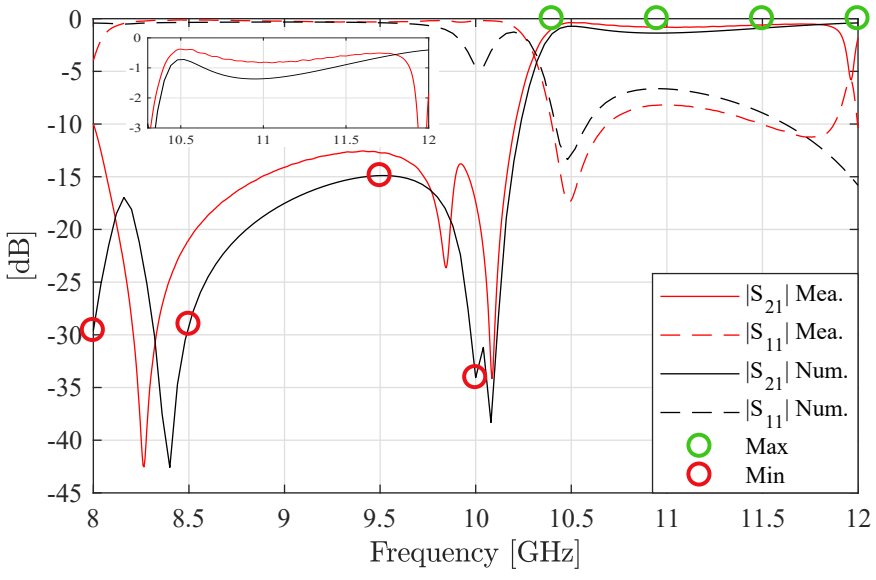

Fig. 13. Comparison between numerical and experimental results for the highpass filter in Figure 12. Maximum measured IL in the bandpass region is $0.8 \mathrm{~dB}$ and minimum rejection outside of the passband is $10.0 \mathrm{~dB}$.

projection methods, with the drawback being a higher insertion loss in the passband. The method provides waveguide filters that can easily be redesigned for specialized applications. It furthermore provides fast prototyping and experimental verification along with easy mass production. We provide examples of filter designs that otherwise could not have been obtained with conventional analytical and geometry based parameter optimization methods. By allowing longer filters in the optimization step, even better filter performances may be obtained.

\section{ACKNOWLEDGMENT}

From Dept. of Electrical Engineering at DTU, the authors would like to thanks Bo Brændstrup for help with PCB manufacturing and Tom K. Johansen for his competences and help at a critical time. We also thank Emilie H. Valente from Dept. of Material Science at DTU for her help with obtaining the micrographs.

\section{REFERENCES}

[1] V. E. Boria and B. Gimeno, "Waveguide filters for satellites," IEEE Microwave Magazine, vol. 8, no. 5, pp. 60-70, 2007.

[2] Y. Konishi and K. Uenakada, "The design of a bandpass filter with inductive stripplanar circuit mounted in waveguide," IEEE Transactions on Microwave Theory and Techniques, vol. MTT22, no. 10, pp. 869-873, 1974.

[3] J. Y. Jin, X. Q. Lin, Y. Jiang, and Q. Xue, "A novel compact e-plane waveguide filter with multiple transmission zeroes," IEEE Transactions on Microwave Theory and Techniques, vol. 63, no. 10, pp. 3374-3380, 2015.

[4] E. Doumanis, G. Goussetis, and J. Huurinainen, "Transmission zero realization in e-plane filters by means of i/o resonator tapping," European Microwave Conference, pp. 767-770, 2016.

[5] G. Goussetis, A. Feresidis, D. Budimir, and J. Vardaxoglou, "Compact ridge waveguide filter with parallel and series-coupled resonators," Microwave and Optical Technology Letters, vol. 45, no. 1, pp. 22-23, 2005.

[6] R. Lopez-Villarroya, G. Goussetis, J. S. Hong, and J. L. GomezTornero, "E-plane filters with selectively located transmission zeros," 2008 European Microwave Conference, Vols 1-3, pp. 733 - 736, 2008.

[7] ESA, "Metal 3d-printed waveguides proven for telecom satellites," 2017. [Online]. Available: https://goo.gl/WXXBpS

[8] J. Bornemann, R. Vahldieck, F. Arndt, and D. Grauerholz, "Optimized low-insertion-loss millimetre-wave fin-line and metal insert filters,"

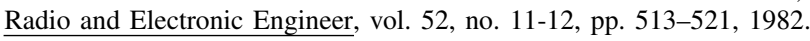

[9] A. Aydoan, F. Akleman, and S. Yldz, "Dielectric loaded waveguide filter design," in 2016 International Symposium on Fundamentals of Electrical Engineering (ISFEE), June 2016, pp. 1-4.

[10] D. Budimir and G. Goussetis, "Design of asymmetrical rf and microwave bandpass filters by computer optimization," IEEE Transactions on Microwave Theory and Techniques, vol. 51, no. 4, pp. 1174-1178, 2003.

[11] R. O. Ouedraogo, E. J. Rothwell, A. R. Diaz, K. Fuchi, and J. Tang, "Waveguide band-stop filter design using optimized pixelated inserts," Microwave and Optical Technology Letters Vol 55, 2013.

[12] N. Aage, E. Andreassen, B. S. Lazarov, and O. Sigmund, "Giga-voxel computational morphogenesis for structural design," Nature, vol. 550, no. 7674, pp. 84-86, 2017.

[13] M. P. Bendsøe and O. Sigmund, Topology optimization Theory, Methods and Applications, 2nd ed. Berlin, Heidelberg, New York: Springer, 2004.

[14] A. Erentok and O. Sigmund, "Topology optimization of sub-wavelength antennas," IEEE Transactions on Antennas and Propagation, vol. 59, no. 1, pp. 58-69, 2011.

[15] E. Hassan, E. Wadbro, and M. Berggren, "Topology optimization of metallic antennas," IEEE Transactions on Antennas and Propagation, vol. 62, no. X, pp. 2488-2500, 2014.

[16] J. Wang, X. S. Yang, X. Ding, and B. Z. Wang, "Antenna radiation characteristics optimization by a hybrid topological method," IEEE Transactions on Antennas and Propagation, vol. 65, no. 6, pp. 28432854, 2017.

[17] A. R. Diaz and O. Sigmund, "A topology optimization method for design of negative permeability metamaterials," Structural and Multidisciplinary Optimization, vol. 41, no. 2, pp. 163-177, 2010.

[18] E. Hassan, D. Noreland, E. Wadbro, and M. Berggren, "Topology optimisation of wideband coaxial-to-waveguide transitions," Scientific Reports, vol. 7, pp. 45 110, 45 110, 2017.

[19] H. Khalil, N. Delhote, S. Bila, M. Aubourg, S. Verdeyme, J. Puech, L. Lapierre, C. Delage, and T. Chartier, "Topology optimization applied to the design of a dual-mode filter including a dielectric resonator," Ieee Mtt-s International Microwave Symposium Digest, pp. 4633 035, 1381-1384, 2008.

[20] N. Aage and V. E. Johansen, "Topology optimization of microwave waveguide filters," International Journal for Numerical Methods in Engineering, vol. 112, no. 3, pp. 283-300, 2017.

[21] K. Svanberg, "The method of moving asymptotes - a new method for structural optimization," International Journal for Numerical Methods in Engineering, vol. 24, no. 2, pp. 359-373, 1987.

[22] N. Aage and B. S. Lazarov, "Parallel framework for topology optimization using the method of moving asymptotes," Structural and Multidisciplinary Optimization, vol. 47, no. 4, pp. 493-505, 2013.

[23] N. Aage, A. Mortensen, and O. Sigmund, "Topology optimization of metallic devices for microwave applications," International Journal for Numerical Methods in Engineering, vol. 83, no. 2, pp. 228-248, 2010.

[24] J. Jin, The finite element method in electromagnetics. John Wiley and Sons Inc., 2002.

[25] Sandia National Laboratories, "Cubit 13.2: geometry and mesh generation toolkit," 2011. [Online]. Available: http://cubit.sandia.gov

[26] G. Karypis and V. Kumar, "A fast and high quality multilevel scheme for partitioning irregular graphs," Siam Journal on Scientific Computing, vol. 20, no. 1, pp. 359-392, 1998.

[27] P. R. Amestoy, I. S. Duff, J. Koster, and J.-Y. L'Excellent, "A fully asynchronous multifrontal solver using distributed dynamic scheduling," SIAM Journal on Matrix Analysis and Applications, vol. 23, no. 1, pp. $15-41,2001$.

[28] P. R. Amestoy, A. Guermouche, J.-Y. L'Excellent, and S. Pralet, "Hybrid scheduling for the parallel solution of linear systems," Parallel Computing, vol. 32, no. 2, pp. 136-156, 2006.

[29] C. A. Balanis, Advanced engineering electromagnetics. Wiley,, 2012.

[30] O. Sigmund, "Morphology-based black and white filters for topology optimization," Structural and Multidisciplinary Optimization, vol. 33, no. 4-5, pp. 401-424, 2007. 


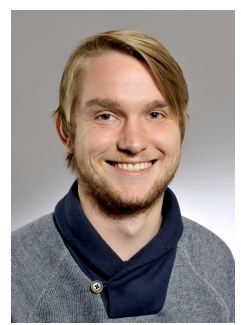

Daniel Gert Nielsen received his M.Sc. degree in Mechanical Engineering, specializing in large scale topology optimization, from the Technical University of Denmark in 2017. After graduation he joined the department of electrical engineering at the Technical University of Denmark as a PhD student. His present research interest include large scale optimization problems, structural optimization and optimization of waveguide filter designs for practical applications.

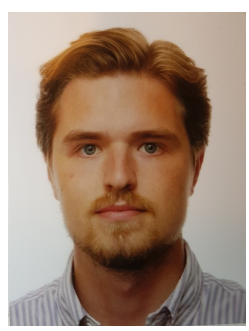

Søren Damgaard Pedersen received his M.Sc. degree in Mechanical Engineering, specializing in large scale topology optimization, from the Technical University of Denmark in 2017. After graduation he proceeded to work with Universal Robots $\mathrm{A} / \mathrm{S}$ developing collaborative robots. His current work concern ongoing optimization of products and processes.

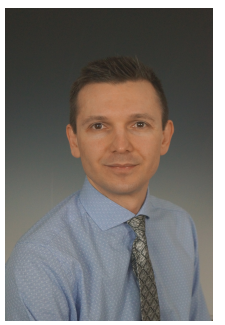

Vitaliy Zhurbenko received his M.Sc. degree from the Kharkiv National University of Radio Electronics in 2001, and the Ph.D. degree from the Technical University of Denmark in 2008, all in Electrical Engineering. In 2005, he joined the Technical University of Denmark, where he is currently an Associate Professor. His present research interests include microwave and millimeter wave sensing for biomedical and security applications; microwave and millimeter-wave components and integrated circuits for instrumentation applications; antennas and passive circuit design and characterization; magnetic resonance technology.

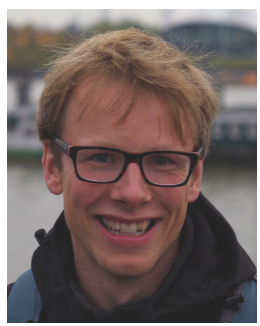

Villads Egede Johansen is a Marie SkłodowskaCurie Fellow (European Commission) at the Department of Chemistry, University of Cambridge, where he investigates natural photonic systems. He obtained his M.Sc. in Electrical Engineering from the Technical University of Denmark (DTU) in 2012, where he worked on topology optimization of microstrip filters. He obtained his Ph.D. on structural colouration of aluminium surfaces from DTU in 2015, which included an external research stay at University of Exeter. His research interests include structural colours, disordered photonics, numerical methods, and topology optimization of electromagnetic problems.

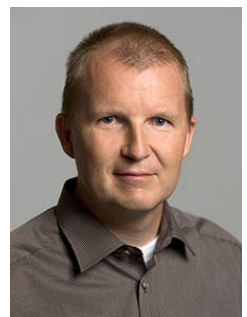

Ole Sigmund is a Professor at the Department of Mechanical Engineering, Technical University of Denmark (DTU). He obtained his Ph.D.- degree 1994 and Habilitation in 2001 and has had research positions at University of Essen and Princeton University. He is a member of the Danish Academy of Technical Sciences and the Royal Academy of Science and Letters (Denmark) and is the former elected President (2011- 15, now EC member) of ISSMO (International Society of Structural and Multidisciplinary Optimization). Research interests include theoretical extensions and applications of topology optimization methods to mechanics and multiphysics problems.

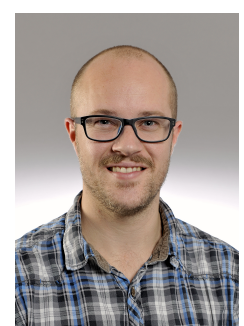

Niels Aage is an Associate Professor at the Department of Mechanical Engineering at the Technical University of Denmark. He received his $\mathrm{PhD}$ in Optimal Design in 2011 from the Technical University of Denmark. His research interest is focused on the area of large scale, parallel numerical methods for PDE constrained optimization, with emphasis on structural optimization problems in multiphysical settings. 